\title{
Mild COVID-19 symptoms despite treatment with teriflunomide and high-dose methylprednisolone due to multiple sclerosis relapse
}

\author{
Nora Möhn ${ }^{1} \cdot$ Firas Saker $^{1} \cdot$ Viktoria Bonda $^{1} \cdot$ Gesine Respondek ${ }^{1} \cdot$ Marcus Bachmann $^{2} \cdot$ Matthias Stoll $^{2}$. \\ Mike P. Wattjes $^{3} \cdot$ Martin Stangel $^{1} \cdot$ Thomas Skripuletz $^{1}$ (])
}

Received: 29 April 2020 / Revised: 13 May 2020 / Accepted: 14 May 2020 / Published online: 28 May 2020

(c) Springer-Verlag GmbH Germany, part of Springer Nature 2020

\section{Dear Sirs,}

The current SARS-CoV-2 pandemic is a huge challenge for health systems around the world. Several risk factors for a severe course of the coronavirus disease (COVID-19) have been identified including advanced age, previous cardiovascular diseases such as diabetes and high blood pressure, male sex, and obesity [1,2]. Since sufficient data are still lacking, it has not yet been possible to clarify whether patients under immunomodulatory therapy are at greater risk [3]. It has been suggested that children and adults with immunosuppression may have a rather favourable disease course compared to the general population [4]. A high-dose steroid treatment generally carries a higher risk of infection $[5,6]$. Hitherto, there is no evidence that coronavirusinfected patients (both in previous outbreaks and currently) with simultaneous steroid therapy have a more severe course of disease $[3,7]$. In the following, we describe the case of a multiple sclerosis (MS) patient who suffered from an undiagnosed SARS-CoV-2 infection and received high-dose methylprednisolone therapy in the assumption of an MS relapse. We show here that the COVID-19 course in this patient was mild and well controllable despite the additional immunosuppressive treatment.

A 42-year old male patient who had been diagnosed with relapsing-remitting MS in 2013 according to the 2010 revisions of the McDonald criteria [8] presented at the emergency room of our clinic in April 2020 due to gait

Thomas Skripuletz

skripuletz.thomas@MH-Hannover.de

1 Department of Neurology, Hannover Medical School, Hannover, Germany

2 Department of Rheumatology and Immunology, Hannover Medical School, Hannover, Germany

3 Department of Diagnostic and Interventional Neuroradiology, Hannover Medical School, Hannover, Germany disturbance which occurred 2 weeks before. Since the initial diagnosis, the MS was sufficiently controlled with teriflunomide. No relapse had occurred so far. He had been clinically asymptomatic, and the annual imaging follow-ups (last in 2019) had not shown inflammatory disease activity. The patient was otherwise healthy. In the context of the current neurological presentation, a mild gait ataxia and hypermetric finger chase test were noticeable. In addition, the patient reported nausea and vomiting, a general weakness and sore throat. Fever or cough had not been noticed. Laboratory analysis showed slightly increased inflammation parameter (Fig. 1a,b). Both, chest X-ray and urine analysis remained unremarkable. The brain MRI showed a new T2 lesion within the right cerebellum (Fig. 1d, previous MRI was performed 6 months before) probably reflecting a symptomatic lesion. As patient's symptoms occurred 2 weeks before, no restriction in diffusion weighted imaging was observable. He was subsequently admitted to the neurological ward and received a high dose methylprednisolone therapy with $1000 \mathrm{mg}$ steroid per day for 4 consecutive days. Neurological deficits completely resolved within 4 days of treatment and consequently the patient wished to be discharged. However, the patient presented at the emergency room the next day with worsening of neurological symptoms. He exhibited fever $\left(>39^{\circ} \mathrm{C}\right)$, hypotension and tachycardia. Serum acute phase reactant (APR) levels further increased (Fig. 1a,b) and an elevated ferritin (1183 $\mu \mathrm{g} / \mathrm{l}$, reference: $27-365 \mu \mathrm{g} / \mathrm{l})$ and Interleukin-6 (29 ng/l, reference $<7 \mathrm{ng} / \mathrm{l})$ were observed 1 day after admission. SARS-CoV-2 was confirmed via RTPCR from nasopharyngeal swabs; the examination for other respiratory viruses (adenovirus, human metapneumovirus, influenza $\mathrm{A} / \mathrm{B}$, parainfluenza, rhinovirus, respiratory syncytial virus) was inconspicuous. Interleukin-6 decreased already after 1 day to a value of $11 \mathrm{ng} / \mathrm{l}$. The patient's condition improved under symptomatic therapy. At rest there was no oxygen requirement. As the fever subsided and the 
A
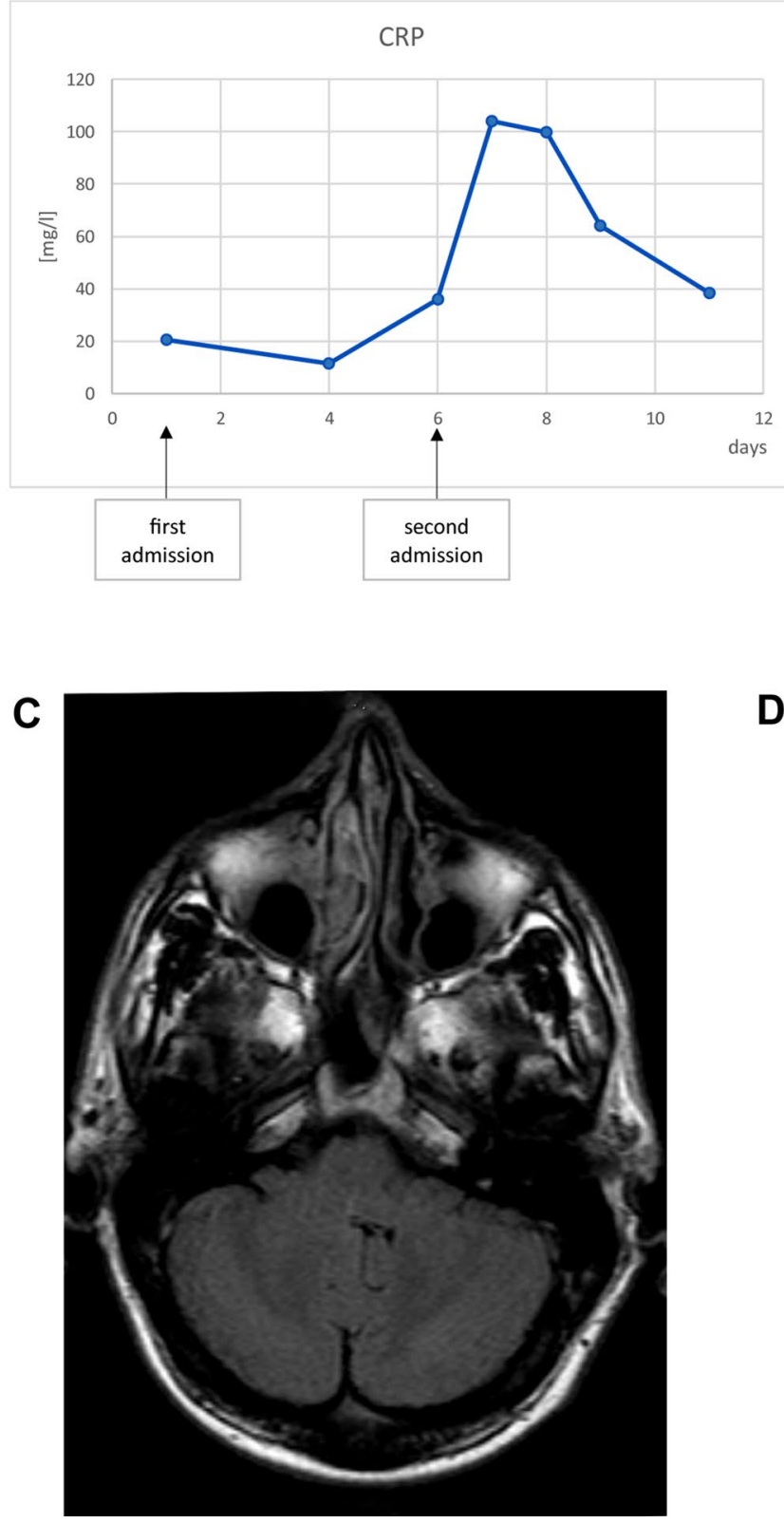

D
B
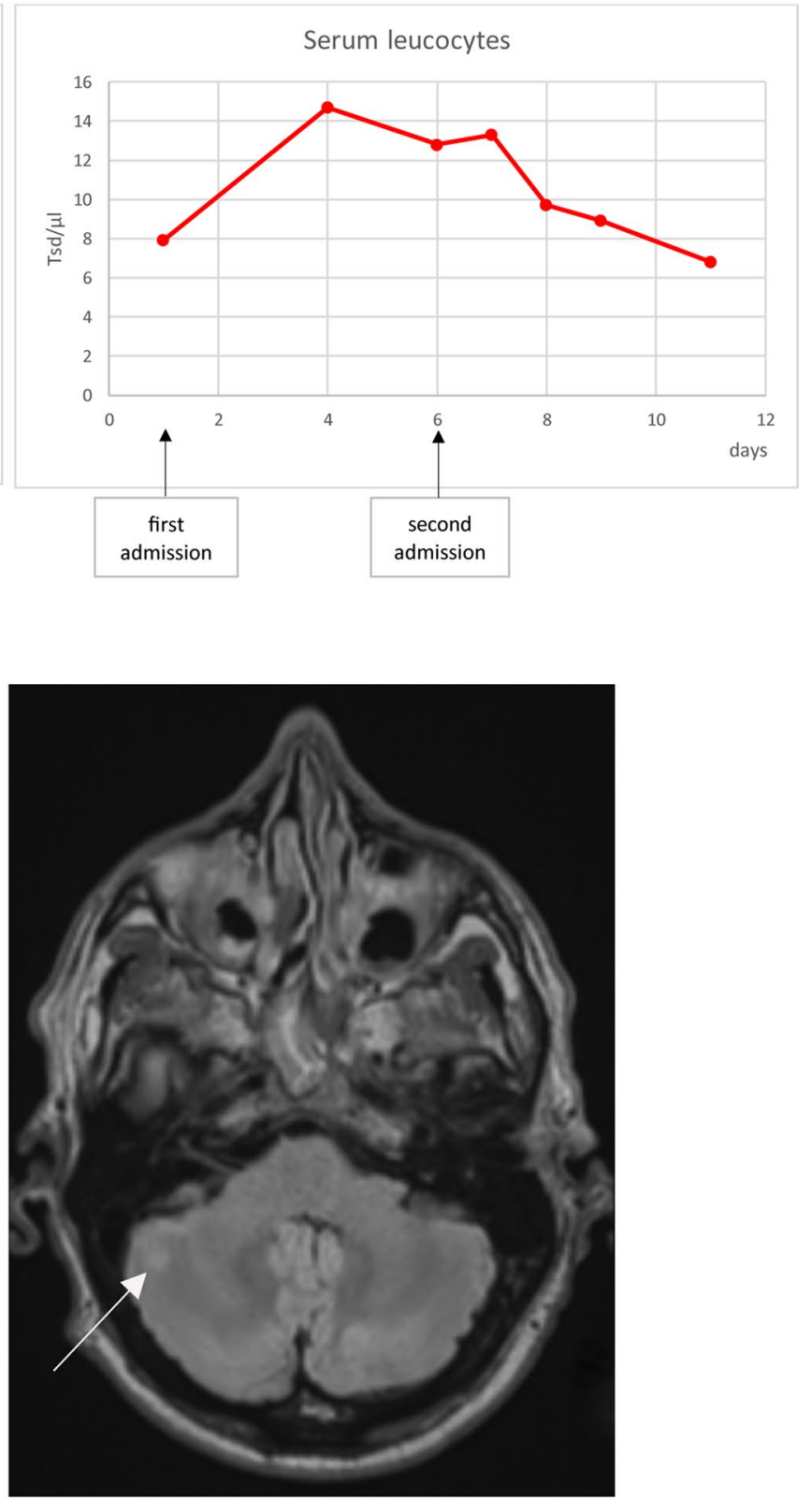

Fig. 1 Course of C-reactive protein (CRP, a) and serum leucocytes (b). Brain MRI imaging, T2-FLAIR. c Brain MRI performed in 07/2013. d Brain MRI performed in 04/2020. The white arrow in d shows the new cerebellar lesion

APR levels dropped significantly, the patient was discharged home after 1 week of inpatient treatment.

In summary, this case shows that a high-dose methylprednisolone pulse therapy did not lead to a severe course of COVID-19 in a male MS patient with pre-existing immunomodulatory therapy with teriflunomide. Due to the incubation period of SARS-CoV-2, it can be assumed that the infection was already present at 1 st admission. A coincidence of a MS relapse and SARS-CoV-2 infection is most likely. The deterioration of neurological symptoms after steroid treatment and the reason for 2 nd admission was rather triggered by the infection, though. Our case of an otherwise healthy man indicates that relapse therapy seems to be safe even in an active undiagnosed SARSCoV-2 infection. However, the extent to which immunosuppressive therapies might increase the risk of a severe disease course will only be clarified in the future when more reliable data are available. 
Funding The manuscript was not funded.

\section{Compliance with ethical standards}

Conflicts of interest The authors declare that they have no conflict of interest.

Ethical standard All investigations and interventions were in accordance with ethical standards.

Consent for publication The patient gave written informed consent for publication.

\section{References}

1. Zheng Z, Peng F, Xu B et al (2020) Risk factors of critical \& mortal COVID-19 cases: a systematic literature review and metaanalysis [published online ahead of print, $2020 \mathrm{Apr} 23$ ]. J Infect. https://doi.org/10.1016/j.jinf.2020.04.021

2. Wang K, Zhang Z, Yu M, Tao Y, Xie M (2020) 15-day mortality and associated risk factors for hospitalized patients with COVID19 in Wuhan, China: an ambispective observational cohort study [published online ahead of print, 2020 Apr 23]. Intensive Care Med. https://doi.org/10.1007/s00134-020-06047-w
3. D“Antiga L (2020) Coronaviruses and immunosuppressed patients: the facts during the third epidemic [published online ahead of print, 2020 Mar 20]. Liver Transpl. https://doi.org/10.1002/ 1t. 25756

4. Minotti C, Tirelli F, Barbieri E, Giaquinto C, Donà D (2020) How is immunosuppressive status affecting children and adults in SARS-CoV-2 infection? A systematic review [published online ahead of print, 2020 Apr 23]. J Infect. https://doi.org/10.1016/j. jinf.2020.04.026

5. Chaudhary NS, Donnelly JP, Moore JX, Baddley JW, Safford MM, Wang HE (2017) Association of baseline steroid use with longterm rates of infection and sepsis in the REGARDS cohort. Crit Care. 21(1):185. https://doi.org/10.1186/s13054-017-1767-1

6. Chanouzas D, McGregor JAG, Nightingale P et al (2019) Intravenous pulse methylprednisolone for induction of remission in severe ANCA associated vasculitis: a multi-center retrospective cohort study. BMC Nephrol. 20(1):58. https://doi.org/10.1186/ s12882-019-1226-0

7. Assiri A, Al-Tawfiq JA, Al-Rabeeah AA et al (2013) Epidemiological, demographic, and clinical characteristics of 47 cases of Middle East respiratory syndrome coronavirus disease from Saudi Arabia: a descriptive study. Lancet Infect Dis 13(9):752-761. https://doi.org/10.1016/S1473-3099(13)70204-4

8. Polman CH, Reingold SC, Banwell B et al (2011) Diagnostic criteria for multiple sclerosis: 2010 revisions to the McDonald criteria. Ann Neurol 69:292-302. https://doi.org/10.1002/ana.22366 